\section{OVERCOMING IMMUNOSUPPRESSIVE TGF- $\beta$ SIGNALING IN HUMAN OVARIAN CANCER-DERIVED TUMOR INFILTRATING LYMPHOCYTES VIA NON-VIRAL CRISPR ENGINEERING}

${ }^{1}$ Samantha Fix*, ${ }^{1}$ Marie-Andrée Forget, ${ }^{1}$ Donastas Sakellariou-Thompson, ${ }^{1}$ Yunfei Wang, ${ }^{1}$ Ana Lucía Dominguez, ${ }^{1}$ Rafet Basar, ${ }^{2}$ Christopher Reyes, ${ }^{2}$ Sanjay Kumar, ${ }^{1}$ Larissa Meyer, ${ }^{1}$ Patrick Hwu, ${ }^{1}$ Chantale Bernatchez, ${ }^{1}$ Amir Jazaeri. ${ }^{1}$ MD Anderson Cancer Center, Houston, TX, USA; ${ }^{2}$ Thermo Fisher Scientific, Carlsbad, CA, USA

Background Our ongoing clinical trial for the treatment of melanoma with TGF- $\beta$-resistant tumor-infiltrating lymphocytes (TIL) $[$ TGF- $\beta$ dominant negative receptor 2 (TGF $\beta D N R 2)$ transduced-TIL] has yielded long-term responses in checkpoint refractory patients (NCT01955460). Building on this success, we sought to extend the impact of TGF- $\beta$-resistant TIL therapy to additional cancers while optimizing a non-viral alternative to transduction with a TGF $\beta D N R 2$. Ovarian cancer $(\mathrm{OvCa})$, which is characterized by an abundance of TGF- $\beta$, a dysfunctional immune infiltrate, and a paucity of novel treatment options, is an ideal candidate for TGF- $\beta$-resistant TIL therapy. Here, we present an optimized and clinically-scalable method for CRISPR/Cas9-mediated deletion of the TGF- $\beta$ receptor (TGFBR2) in OvCa TIL.

Methods OvCa TIL were generated from tumor fragments ${ }^{1}$ and subjected to CRISPR-mediated knockout of TGFBR2 before going through a rapid expansion protocol. Resistance of TGFBR2-knockout TIL to TGF- $\beta$ signaling was evaluated via quantification of downstream SMAD-2/-3 phosphorylation, global transcriptional changes upon TGF- $\beta$ exposure, and cytokine release following TCR stimulation in the presence of TGF- $\beta$. The impact of CRISPR modification on TIL expansion and TCR clonal diversity was evaluated. Finally, the risk of off-target CRISPR activity throughout the genome was evaluated using Target Enriched GUIDE-seq (TEG-seq) ${ }^{2}$ followed by next generation sequencing (NGS) validation of putative off-target sites.

Results Using five TGFBR2-directed guide RNAs (gRNAs), we achieved gene disruption efficiencies ranging from 48\%-90\%, which correlated inversely with the degree of SMAD phosphorylation after TGF- $\beta$ exposure $(r=-0.9440, \quad p=0.0158$, $\mathrm{n}=4$ donors) (figure $1 \mathrm{~A}-\mathrm{C}$ ). TGF- $\beta$ exposure induced a strong transcriptional response in wild-type TIL but had little to no effect on TGFBR2-knockout TIL (figure 2). TGFBR2-knockout TIL functioned well in the presence of exogenous TGF- $\beta$ as evidenced by equally strong secretion of pro-inflammatory cytokines in the presence and absence of TGF- $\beta$ (figure 3). CRISPR-modification did not hamper the ex vivo expansion efficiency nor the TCR clonal diversity of expanded OvCa TIL (figure 4). Using TEG-seq, we identified $\leq 5$ low-probability off-target sites for gRNA-\#3 and gRNA-\#4, each of which were attributed to background sequencing artifacts upon further validation by NGS of specific amplicons (figure 5).

Conclusions CRISPR/Cas9-mediated knockout of TGFBR2 is feasible and efficient in patient-derived OvCa TIL using clinically-scalable methods that yield little to no evidence of offtarget activity. This study lays the groundwork for clinical translation of CRISPR-modified, TGF- $\beta$-resistant TIL for OvCa treatment, which will not only provide a novel immunotherapy for $\mathrm{OvCa}$ patients but also a platform for engineering more potent TIL therapies in general.

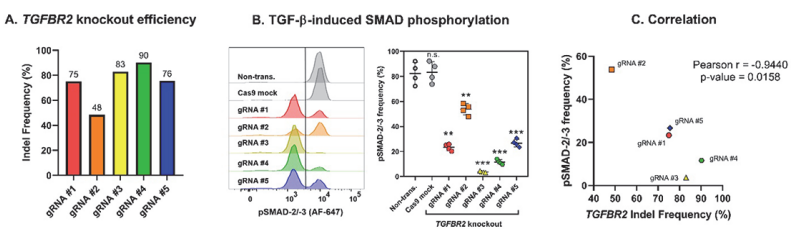

Abstract 172 Figure 1 (A) Genomic-level TGFBR2 knockout efficiency using 5 different gRNAs as evidenced by NGS of specific amplicons ( $n=1$ TIL donor). (B) SMAD-2 and SMAD-3 phosphorylation in TGFBR2 knockout TIL vs. control TIL after 30 min exposure to TGF- $\beta 1$. The left panel shows representative histograms of phospho-SMAD staining, and the right panel shows quantification of cells positive for phosphoSMAD-2/-3 after TGF- $\beta$ exposure ( $n=4$ TIL donors). The statistical significance of each experimental condition compared to the nontransfected control is shown. (C) Inverse correlation of TGFBR2 knockout efficiency and TGF- $\beta$-mediated SMAD phosphorylation.

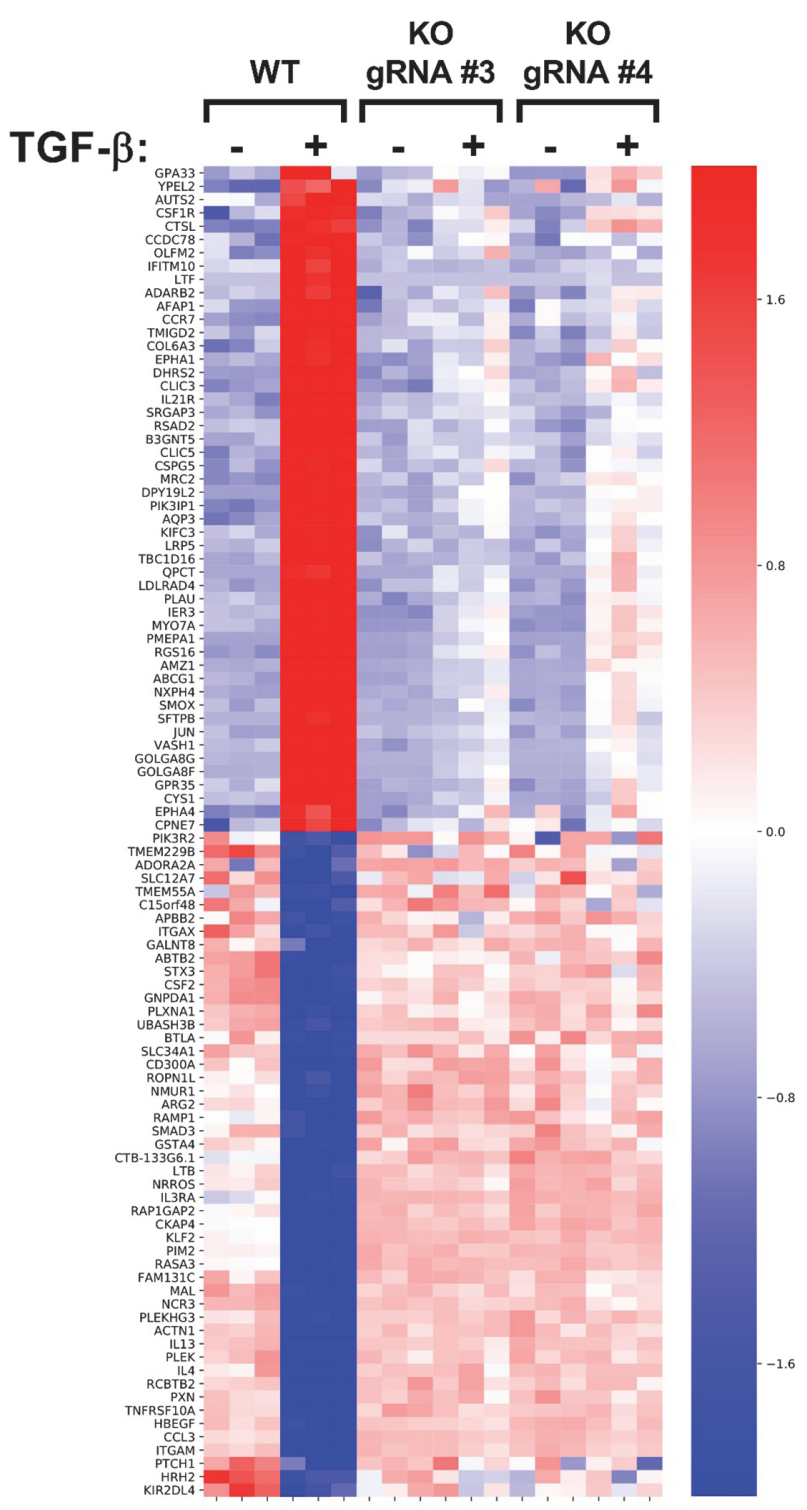

Abstract 172 Figure 2 Top 100 differentially expressed genes in nontransfected (WT) TIL exposed to TGF- $\beta$. TGFBR2 knockout (KO) TIL display minimal gene expression changes upon TGF- $\beta$ exposure $(n=3$ technical replicates). 


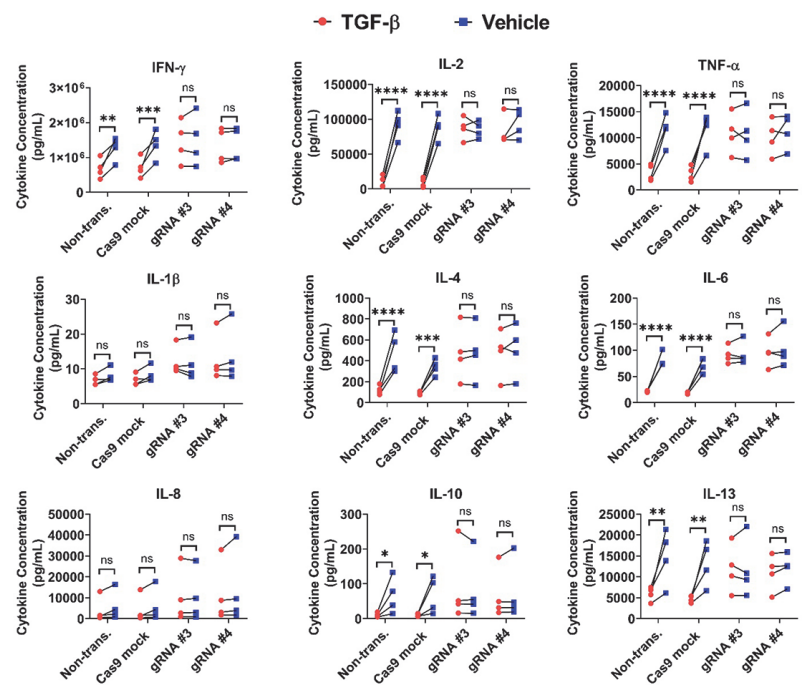

Abstract 172 Figure 3 TIL were collected after 14 days of expansion and re-stimulated with $300 \mathrm{ng} / \mathrm{mL}$ plate-bound anti-CD3 in the presence of $3000 \mathrm{IU} / \mathrm{mL}$ IL2 and $10 \mathrm{ng} / \mathrm{mL}$ human TGF- $\beta 1$ or vehicle. Cell culture supernatant was collected after $72 \mathrm{hrs}$ of stimulation and assayed for the presence of 10 proinflammatory cytokines (IFN- $\gamma$, IL-1 $\beta$, IL-2, IL-4, IL-6, IL-8, IL-10, IL-12p70, IL-13, and TNF- $\alpha$ ). For TIL with intact TGFBR2 (non-transfected and Cas9 mock transfected TIL), the production of many pro-inflammatory cytokines decreased significantly in the presence of TGF- $\beta$. Conversely, TGFBR2 knockout TIL (generated using gRNA \#3 or gRNA \#4) retain cytokine secretion in the presence of TGF- $\beta$. IL-12p70 was below the limit of detection in this assay and is therefore not presented.

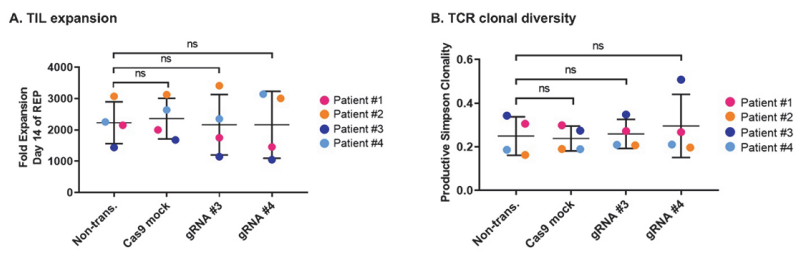

Abstract 172 Figure 4 (A) Control and CRISPR-modified OvCa TIL expand with equal efficiency during a 14-day rapid expansion protocol. Fold expansions ranging from 1000x - 3000x were observed across 4 independent patient samples. (B) The TCR clonal diversity of TIL after 14-day expansion was assessed by TCRB sequencing. Productive Simpson Clonality was equivalent in CRISPR-modified TIL compared to control TIL samples.

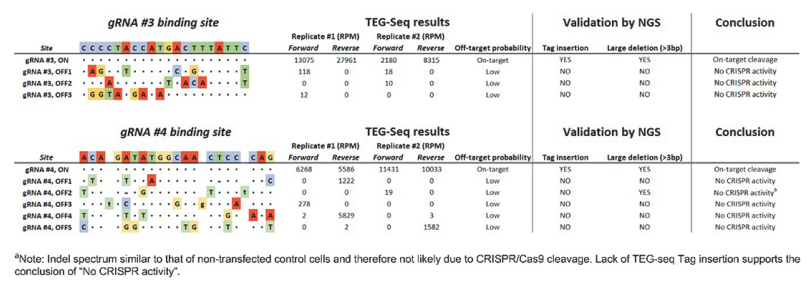

Abstract 172 Figure 5 TEG-seq revealed 3 putative off-target sites for gRNA \#3 and 5 putative off-target sites for gRNA \#4. The aligned sequences show similarities and differences between the gRNA sequence and the reference genome site. Dots represent exact matches in the reference genome compared to the gRNA sequence. Dashes represent missing bases, lower-case letters represent extra bases, and upper-case letters represent a base mismatch. Validation by NGS of specific amplicons confirmed the presence of TEG-seq Tag integration and large indels at the on-target cleavage sites for gRNA \#3 and \#4, indicating successful Cas9 editing and Tag integration in our experiment. NGS validation revealed that all putative low probability off-target sites were background artifacts as evidenced by the lack of Tag identification and lack of large indels.

\section{REFERENCES}

1. Sakellariou-Thompson D, Forget MA, Hinchcliff E, Celestino J, Hwu P, Jazaeri AA, et al. Potential clinical application of tumor-infiltrating lymphocyte therapy for ovarian epithelial cancer prior or post-resistance to chemotherapy. Cancer Immunology, Immunotherapy: CII 2019;68(11):1747-57.

2. Tang PZ, Ding B, Peng L, Mozhayskiy V, Potter J, Chesnut JD. TEG-seg: an ion torrent-adapted NGS workflow for in cellulo mapping of CRISPR specificity. Bio Techniques 2018;65(5):259-67.

Ethics Approval All procedures performed were in accordance with the 1975 Helsinki declaration. Ethical approval and tissue from surgical resections used to expand TIL were both obtained under protocols (PA16-0912 and LAB02-188) approved by the Institutional Review Board of The University of Texas MD Anderson Cancer Center. Written informed consent was obtained from all individual participants included in the study for their specimens and data to be used in research and for publication.

http://dx.doi.org/10.1136/jitc-2021-SITC2021.172 